\title{
Compact mmWave FMCW Radar: Implementation and Performance Analysis
}

\author{
Seifallah Jardak* ${ }^{*}$ Tero Kiuru ${ }^{\dagger}$, Mikko Metso ${ }^{\dagger}$, Sajid Ahmed ${ }^{\ddagger}$, and Mohamed-Slim Alouini* \\ * King Abdullah University of Science and Technology, Makkah Province, Saudi Arabia, \\ seifallah.jardak@kaust.edu.sa \\ $\dagger$ VTT Technical Research Centre of Finland Ltd., Espoo, Finland \\ $\ddagger$ Information Technology University, Lahore, Pakistan.
}

\begin{abstract}
The evolution of single-chip high-frequency circuits helped manufacture radars with better accuracy, lower costs, and smaller footprints. This paper describes a compact $24 \mathrm{GHz}$ frequency-modulated-continuous-wave radar that can be used to aid the visually impaired during their outdoor journeys. The project helps partially or totally blind people avoid collision with their environment without the use of a white cane. Details about the implemented signal processing algorithms are also given. Although its main purpose is to assist the visually impaired, the device can perform diverse tasks such as rangedirection mapping, velocity estimation, presence detection, and vital sign monitoring. The experimental result section demonstrates the device's capabilities through different use-cases.
\end{abstract}

Index Terms-Chirp radar, continuous-wave (CW) radar, frequency-modulated continuous wave (FMCW) radar, linear FMCW (LFMCW), millimeter wave, slowtime processing, low-bandwidth, civil applications.

\section{INTRODUCTION}

D UE to an increasing demand in channel capacity and bandwidth, there has been a growing interest in the millimeter wave (mmWave) spectrum [1]-[3]. Many researchers overcame the challenges of implementing extremely high frequency systems and secured large bandwidths comparable to that of fiber optics [4]. The mmWave spectrum offers numerous advantages not only in the wireless communication field but also in the field of sensors and radar technology [5]. Indeed, transmitting shorter wavelengths helps radars achieve higher range accuracy with smaller antenna footprints.

Drawn by the strong prospects of the mmWave band, the semiconductor industry followed the trend [6] and manufactured a panoply of integrated radar-on-chips to reduce the system design cost and complexity. This contributed to a significant growth of radar based solutions for different civil applications [7]. The flagship example is the automotive industry with its advanced features like adaptive cruise control and collision avoidance systems.
Additionally, radars are now found in areas like building security [8], industry, civil engineering or even health care [9]-[11].

The linear frequency-modulated continuous wave (FMCW) radar transmits frequency modulated signals to determine the range of targets [12]. Its resolution is inversely proportional to the signal's bandwidth $\beta$ and expressed as $\Delta R=\frac{c}{2 \beta}$, where $c$ is the speed of light. The transmitted pulse is reflected from a target located at range $R$ and received after a round trip timedelay $t_{d}=\frac{2 R}{c}$. This delay is measured by comparing the received frequency with the frequency of the local oscillator. Assuming low target velocity, the frequency shift, also called beat frequency $f_{b}$, is easily derived as $f_{b}=\frac{\beta}{\tau} t_{d}=\frac{2 R \beta}{\tau c}$ where $\tau$ is the pulse duration.

The voltage-control-oscillator (VCO) is a key component in high frequency systems. They differ by the carrier frequency, the maximum allowed bandwidth and the number of transmit and receive antennas. The $24 \mathrm{GHz}$ frequency band is commonly used for short-range radar applications, while the $76-77 \mathrm{GHz}$ band is used in midand long-range applications. The literature discusses different approaches for implementing a mmWave radar system. An elaborate design employs a commercial VCO to build a $24 \mathrm{GHz}$ FMCW radar with multiple bandwidths ranging from $200 \mathrm{MHz}$ up to $2 \mathrm{GHz}$ [13]. To store and process the received data in real time, the back-end module is based on a field-programmablegate array (FPGA) and a digital signal processor (DSP). This configuration enhances the radar's capabilities at the expense of cost and portability. Another recent work [14] aims to sense hand gestures at close range using monopulse FMCW radar. The design is based on two commercial VCOs connected to one transmit and three receive antennas. Despite the VCO's $250 \mathrm{MHz}$ bandwidth limitation, the system radiates frequencies ranging from 22.5 to $26.5 \mathrm{GHz}$, resulting in $4 \mathrm{GHz}$ bandwidth. Therefore, a chirp linearization technique is necessary to compensate for the highly non-linear voltage-frequency 
response.

This work describes a portable $24 \mathrm{GHz}$ linear FMCW radar, designed to assist the visually impaired during their outdoor journeys. Guidesense [15] is a wearable assistive device destined for partially or totally blind users to avoid collision with people or human sized obstacles. During clinical trials, users felt that Guidesense improved their confidence in independent mobility and orientation [16]. As shown in Fig. 1, the compact design can fit in a $10 \times 4 \times 2 \mathrm{~cm}^{3}$ box and weighs less than 150 grams. The radar front-end module and patch antennas are printed on a 4 layer PCB and mounted on top of a commercial micro-controller board. Next, the signal processing algorithms programmed inside the microcontroller are discussed. Besides obstacle detection, the prototype can fulfill multiple purposes, such as velocity estimation, range-direction mapping, and vital sign (breathing) monitoring.

\section{HARDWARE DESCRIPTION}

The radar's hardware design is described in this section. The hardware modules are grouped in the following subsections based on the system frequency and data type.

\section{A. Linear FMCW Waveform Generation}

A basic block diagram of the designed FMCW radar is presented in Fig. 2. A phase-locked-loop (PLL) circuit drives the VCO to perform a frequency sweep. In this work, the Infineon radar chip BGT24MTR12 is used with one transmitter and two in-phase and quadraturephase (IQ) receivers. During a pulse duration $\tau=250 \mu \mathrm{s}$, the VCO emits a linear chirp signal with maximum transmit power of $11 \mathrm{dBm}$ and frequency varying between 24 and $24.25 \mathrm{GHz}$. The process is repeated at each pulse repetition interval (PRI).

The PLL circuit ADF4158 minimizes the phase error between the VCO's feedback and the reference signal

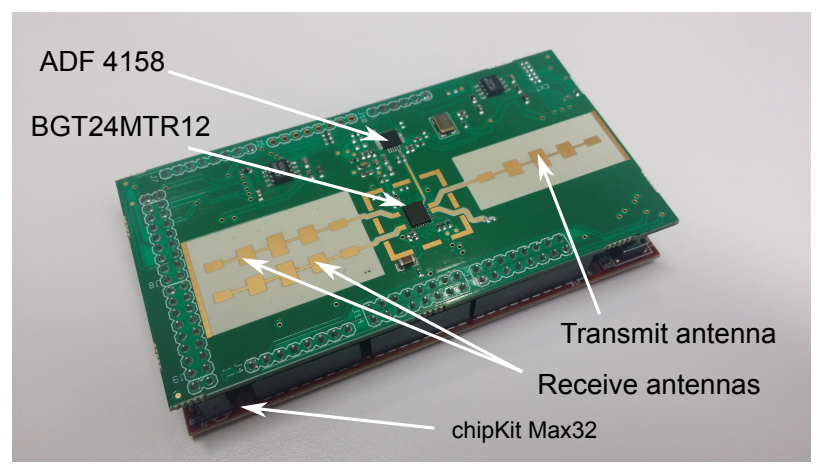

Fig. 1. A compact short-range linear FMCW radar with one transmit and two receive antennas. and generates a stable high frequency output. The PLL's charge pump passes by an external loop filter before going in the VCO's coarse input. The third-order loop filter, designed in ADIsimPLL software [18], [19], ensures a root mean square frequency deviation of $4.14 \mathrm{kHz}$ from the desired perfectly linear up-ramp.

\section{B. Antenna Design}

Equipped with two receive antennas, the radar resolves targets located in a single plane. If an echo is reflected from a different plane, the radar will correctly estimate its range but not its direction. To alleviate this source of ambiguity, the antenna's beamwidth should be narrow in azimuth and wide in elevation angles. For this reason, a patch antenna array of 5 rectangular microstrip elements is engineered as illustrated in Fig. 3. The amplitudes of the side elements are tapered to minimize the far field radiation sidelobes [20], [21]. Figure 4 shows the azimuth radiation pattern simulated on a $0.254 \mathrm{~mm}$ thick Ro4350B substrate using ANSYS High Frequency Electromagnetic Field Simulation (HFSS). This antenna setting achieves a $3 \mathrm{~dB}$ beamwidth of $65^{\circ}$ in elevation and $26^{\circ}$ in azimuth. The first far field sidelobes occur at angles $40^{\circ}$ and $-68^{\circ}$ with levels less than $-22 \mathrm{~dB}$. The graph of the antenna reflection coefficient $S_{1,1}$ as function of the transmitted frequency is plotted in Fig. 5. Within the range of interest, the $S_{1,1}$ parameter is less than $-11.5 \mathrm{~dB}$ and reaches its minimum value $-16.11 \mathrm{~dB}$ at $24.15 \mathrm{GHz}$. This translates in a maximum realized gain of $11.4 \mathrm{~dB}$. The VCO provides a differential radio frequency (RF) output. However, the radar

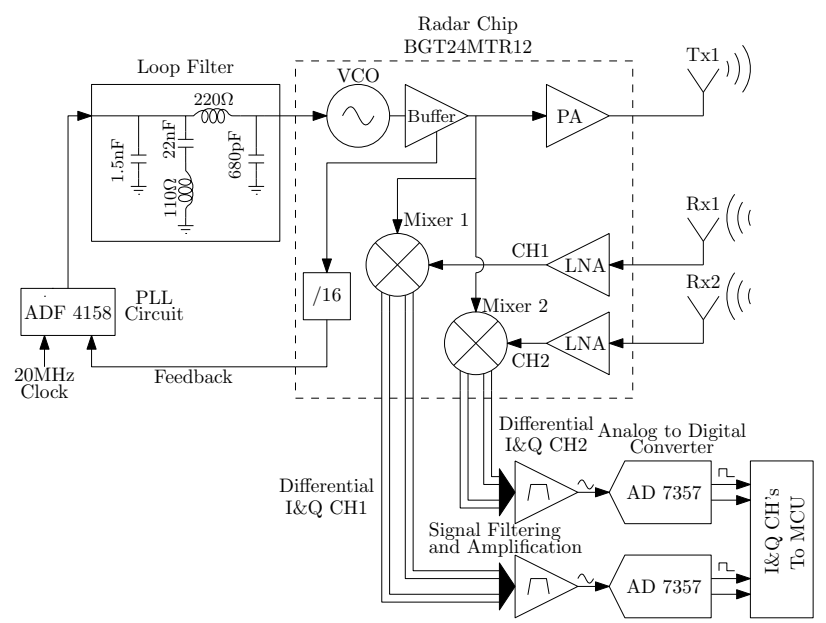

Fig. 2. Diagram of the radar's analog hardware design. Using a four layer PCB, the high frequency RF components are grouped on top layer, while active amplifiers and ADC converters are printed in bottom layer for extra RF shielding. The detailed architecture of the radar chip is given in [17]. 


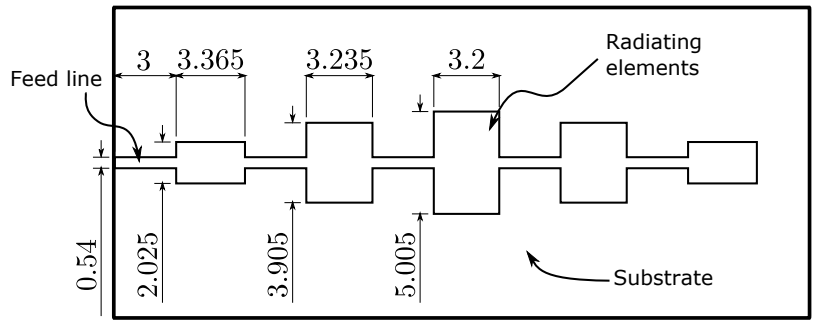

Fig. 3. Illustration of the 5 element antenna array used for transmission and reception. The substrate is a $0.254 \mathrm{~mm}$ thick Ro4350B roger board.

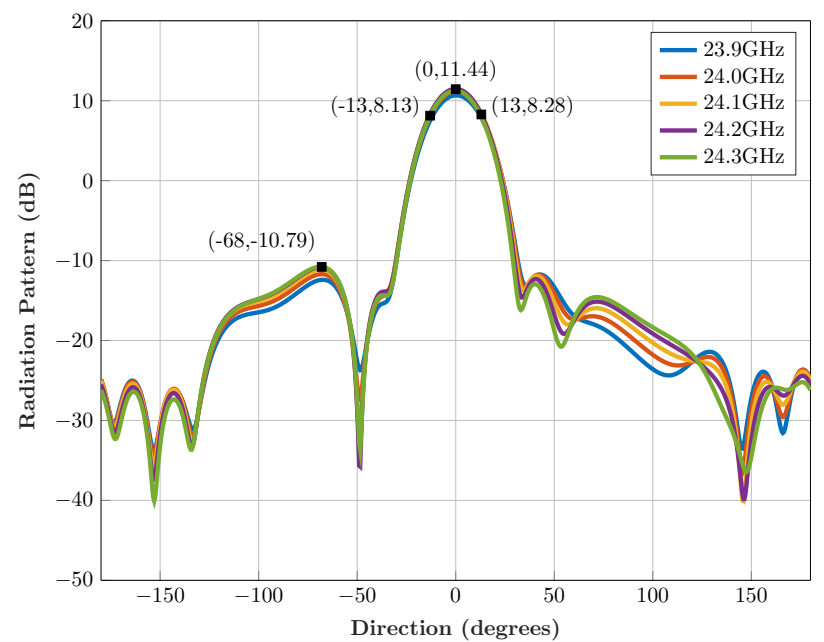

Fig. 4. Far field azimuth radiation pattern of the 5 element array simulated at different frequencies from $23.9 \mathrm{GHz}$ to $24.3 \mathrm{GHz}$ with $100 \mathrm{MHz}$ step.

is equipped with only one transmit antenna to keep the effective isotropic radiated power (EIRP) within the ISM band limitation, i.e. $20 \mathrm{dBm}$. The second RF output is terminated with a $50 \Omega$ resistor.

\section{Signal Amplification and Filtering}

The received signals from both receive antennas are down converted inside the BGT radar chip and outputted as four pairs of differential I/Q signals. The differential signals reduce the electromagnetic interference and improve noise immunity. Next, each wire passes by three stages of inverting band-pass filters. The active filters not only amplify the signal but also attenuate its low frequency components originated from insufficient isolation between the transmit and receive antennas (i.e the leakage from the transmitter resembles a close range reflection to the receiver). In addition, the $60 \mathrm{~dB} / \mathrm{decade}$ roll-off compensates for the power attenuation caused by the quartic term $1 / R^{4}$ in the radar equation.

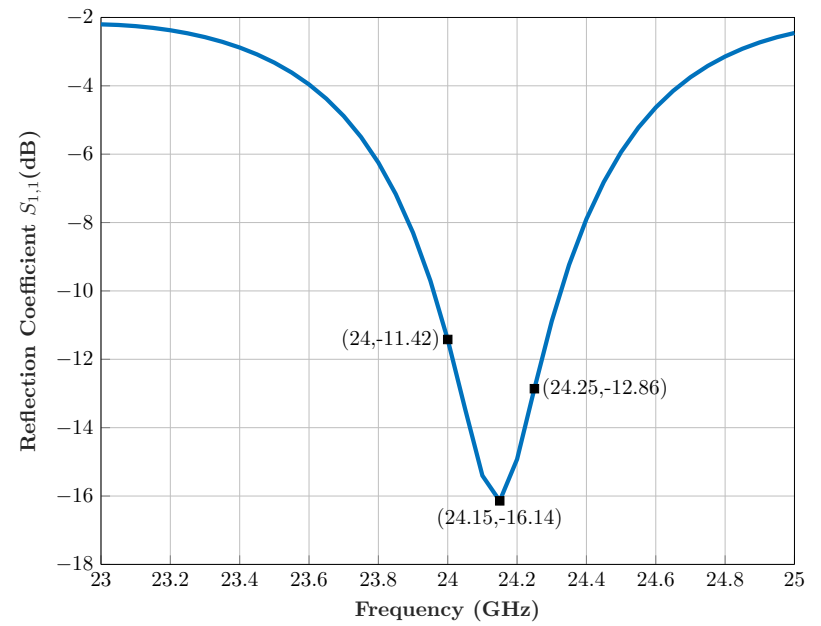

Fig. 5. The reflection coefficient $S_{1,1}$ of the 5 element antenna array simulated using HFSS software.

\section{Data Sampling}

Two AD7357 circuits are used to sample the data. Each circuit contains dual, 14-bit analog to digital converter (ADC) with differential input. Then, the sampled data is transmitted through the serial-peripheral-interface (SPI) to the micro-controller PIC32MX795F512L, which is part of the chipKit Max32 development kit. The microcontroller runs at $80 \mathrm{MHz}$ with $128 \mathrm{kB}$ data memory.

To configure the radar's modes of operation and plot the processed data, the micro-controller is connected through USB UART to a PC. The latter runs a python code which continuously parses and plots the received data. The radar system is powered by a $5.0 \mathrm{~V}$ source and consumes approximately $400 \mathrm{~mA}$. When connected to a PC, the radar can use the USB interface for both power and data transmission.

In the next section, we will present the signal processing modules implemented to operate the portable radar device.

\section{SOFTWARE IMPLEMENTATION}

An overview of the signal processing blocks is shown in Fig. 6. The code is developed in C and fits in $200 \mathrm{kB}$, corresponding to roughly $39 \%$ of the micro-controller's program memory.

Depending on the maximum detectable speed required by the application, the ADF4158 delay register sets the desired PRI, then generates the continuous sawtooth waveform. Immediately, the ADC starts sampling the received baseband signals at rate $f_{s}$. The sampling rate is maintained constant using a timer interruption. Following the Nyquist theorem, the maximum detectable beat frequency is $f_{b, \max }=\frac{f_{s}}{2}$, which translates in a maximum 


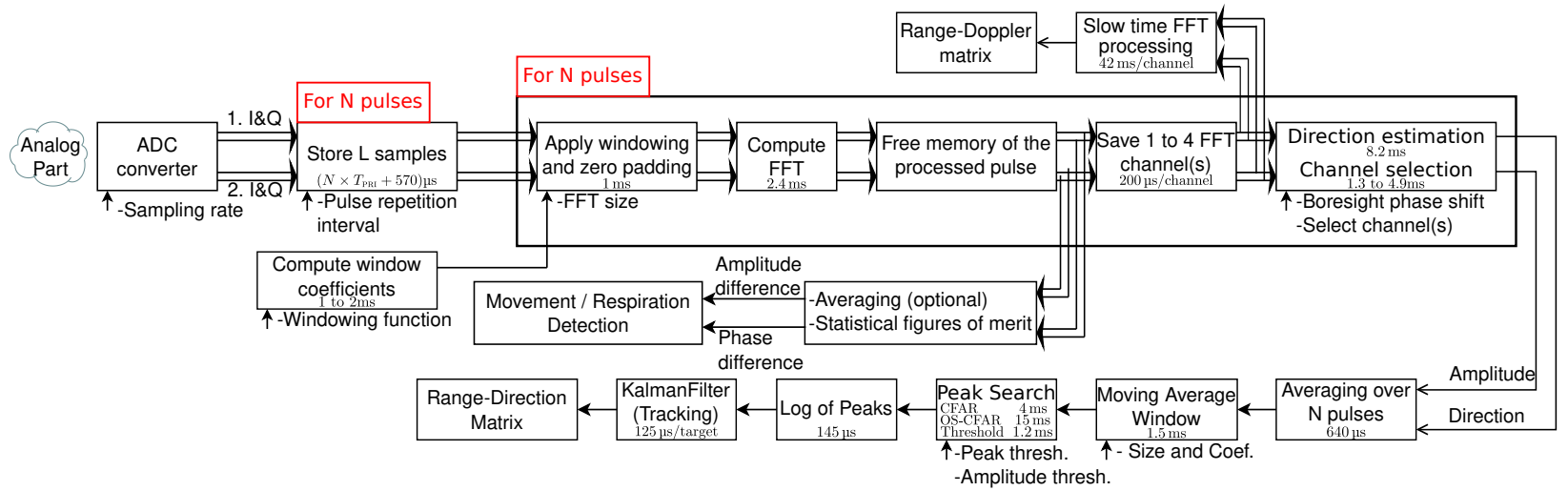

Fig. 6. Diagram of the radar's signal processing modules. The average execution time is computed for 125 raw data samples and an FFT size equal to 512 .

reachable range $R_{\max }=\frac{c f_{s} \tau}{4 \beta}$. In each sampling clock, 4 raw data are stored in a dynamically allocated memory. The samples correspond to the I and Q channels of both receivers. In total, $L=4 \tau f_{s}$ samples are stored during a single pulse and the values sampled between pulses are discarded. Each raw data sample is stored in 2 bytes.

Multiple coherent pulses are stored to perform slow time processing and estimate target's velocity. The maximum detectable radial velocity is inversely proportional to PRI [22], [23] and expressed as $V_{\max }=\frac{c}{4 f_{c} T_{\mathrm{PRI}}}$. Besides, the number of pulses per scan $N$ affects the velocity resolution $\Delta V$ through the relationship $\Delta V=$ $\frac{2 V_{\max }}{N}=\frac{c}{2 f_{c} N T_{\mathrm{PRI}}}$.

Once the raw data matrix is filled, a windowing function is applied to reduce the sidelobes caused by spectral leakage and multi-reflections. Although this technique brings minor improvements in an outdoor open environment, it is essential when multi-reflections are considerable, for instance during indoor target detection. Next, for each pulse, the fast Fourier transform (FFT) of the four channels is computed. The interferometry technique [24], [25] determines the phase difference between the signal received at the first and second antennas to estimate the direction of arrival of echos. Ideally, a target located at the radar's boresight indicates a zero phase difference. However, due to different wire lengths in the hardware design, a constant non-null phase shift is detected. To avoid phase wrapping around the radar's boresight, the phase difference is centered and shifted back to zero.

At this stage, for better memory management, the raw data of the processed pulse is released and a new memory slot is dynamically allocated to store the complex FFT result. Each complex FFT sample is stored in a structure of 8 bytes. Once all $N$ pulses are processed, the remaining signal processing modules are grouped in three major blocks based on the following modes of operation.

\section{A. Range-Direction Map}

First, the average of the $N$ amplitudes and directions is computed, then, a simple moving average window is applied to smooth the vector of amplitudes. Due to radar's limited bandwidth and wide target mainlobes, the performance of constant false alarm rate (CFAR) based methods deteriorates when the reference cells are contaminated by nearby targets. As a result, a simpler peak search algorithm is implemented. A local maximum is considered a potential target when its amplitude is greater than $\Delta_{A}$, and the difference between the peak and its valleys is higher than $\Delta_{T}$. The routine outputs a vector of boolean bits to indicate the presence of a peak at each FFT range bin. During experimental measurements, the thresholds $\Delta_{A}$ and $\Delta_{T}$ are respectively set to $15 \mathrm{~dB}$ and $3 \mathrm{~dB}$.

During field tests, it was noticed that the instantaneous peak information is not stable. The radar may miss a target during few sweeps due to fluctuations in the target's radar cross section as it moves and turns relative to the receiver. Moreover, in indoor applications, high sidelobes are often mistaken for false targets. To identify and track multiple targets, it is crucial to properly associate the radar measurements with their corresponding tracked targets. Thus, a logging method is implemented to record a history of peak occurrences over successive scans. The method analyses the current vector of peaks generated by the search algorithm and compares it with its log table. If a new peak is detected within a guard bin $\Delta_{\mathrm{G}}$ from a previously logged peak, the log table is updated. It records the peak's new location and increments its number of occurrences. If the latter exceeds $N_{\text {OC }}$ during the recent $N_{\mathrm{T}}$ scans, the radar identifies the peak as a target and triggers a Kalman filter to track it. Besides, if a logged peak is currently not detected, its peak location is set to a default value, i.e. zero, and the log table notes that the peak is missed. A logged peak is excluded from 


\begin{tabular}{|c|c|c|c|c|}
\hline \multirow[t]{2}{*}{ Peak search } & \multicolumn{2}{|c|}{$\begin{array}{l}\text { Incorporate current peak } \\
\text { information in the log table }\end{array}$} & \multicolumn{2}{|r|}{ Maintain the log table } \\
\hline & $\begin{array}{l}\text { - Left shift } V_{o c} \\
\text { - Test: Range } \\
\Delta_{G} \text { from pre }\end{array}$ & $\begin{array}{l}=V_{o c}(i)<<1 \\
\text { new peak within } \\
\text { usly logged peak }\end{array}$ & False & $\begin{array}{l}\text { - Merge two entries less than } \Delta_{\min } \text { away } \\
\text { - Evaluate } \dot{N}_{o c} \text { and } \dot{N}_{\text {DROP }} \text { using a relevant } \\
\text { bitwise sum of } V_{o c} \\
\text { - Test } \dot{N}_{o c} \geq N_{p c} \text { : } \\
\text { - Assign a Kalman filter for tracking }\end{array}$ \\
\hline \multicolumn{2}{|c|}{$\begin{array}{l}\text { - Insert new entry in log table } \\
\text { - Store current range and direction } \\
\text { Set } V_{o c}=1\end{array}$} & $\begin{array}{l}\text { - Update previc } \\
\text { - Store current } \\
\text { - Update } V_{o c}=\end{array}$ & $\begin{array}{l}\text { us entry in log table } \\
\text { cange and direction } \\
V_{o c}+1\end{array}$ & $\begin{array}{l}\text { - Test } N_{\text {DROP }} \geq N_{\text {DROP }} \\
\text { - Delete entry from log table } \\
\text { - Free asigned Kalman filter }\end{array}$ \\
\hline
\end{tabular}

Fig. 7. List of steps used to log and track the instantaneous peaks.

the table only if it is missed during $N_{\text {DROP }}$ successive scans. Finally, if the distance between two logged peaks is smaller than $\Delta_{\text {min }}$, the two entries are merged. Hence, the first $T$ targets correspond to the first $T$ logged peaks with more than $N_{\mathrm{OC}}$ occurrences during the last $N_{\mathrm{T}}$ scans. For implementation, the length of the log table is set to $5 T$. Next, multiple Kalman filters are used to track the first $T$ targets of interest and smooth their rapidly changing direction information. Fig. 7 present a flowchart illustrating the different steps used to log and track the instantaneous peaks.

At last, three vectors, i.e. signal amplitudes, direction and peak information, are transmitted to the PC's python code to plot the range-direction map.

\section{B. Range-Doppler Map}

The slow time processing estimates the target's Doppler shift and helps separate two close targets moving with different speeds. After computing the fast time FFT, a second FFT operation is applied on the slow time data. The number of pulses $N$ should be at least 16 , as it corresponds to the smallest FFT size implemented in the XC32 DSP library. The minimum achievable PRI is $T_{\mathrm{PRI}}=300 \mu \mathrm{s}$, which translates in a maximum detectable speed $V_{\max }=37.3 \mathrm{~km} \cdot \mathrm{h}^{-1}$.

Due to its limited memory, the micro-controller is able to store and process $N=32$ pulses per scan. To increase this number and improve the Doppler resolution, the slow time processing is applied over a specified range of interest. Hence, by only storing the required FFT range bins, the method can be carried out with $N=64$ pulses per scan.

\section{Respiration and Movement Detection}

The third and last mode of operation senses respiration and movement inside a room [26]. It compares the statistical fluctuation of the signal's amplitude and phase to reliably identify if a person is breathing, holding his breath or moving in front of the radar unit.

To identify breathing movements, the phase of the received signal is analyzed. A change in phase, also referred as micro-Doppler, is proportional to movements which are in the order of the signal's wavelength, i.e. $\lambda=\frac{c}{f_{c}} \approx 12.5 \mathrm{~mm}$, and it can be expressed as follows

$$
\Delta \phi_{i, c}=\min \left\{\left|\phi_{i, c}^{\text {current }}-\phi_{i, c}^{\text {ref }}\right|, 360-\left|\phi_{i, c}^{\text {current }}-\phi_{i, c}^{\text {ref }}\right|\right\},
$$

where $\phi_{i, c}^{\text {current }}$ is the current instantaneous phase at channel $c$ and range bin $i$ and $\phi_{i, c}^{\text {ref }}$ is a reference phase. The reference phase can be the previous phase or an average of $k$ previous phases. The squared mean of the phase difference over a set of range bins is used as an indicator of breathing movements and is written as

$$
\phi_{\mathrm{MS}}=\frac{\sum_{i=i_{\text {start }}}^{i_{\text {stop }}} \min _{c}\left(\Delta \phi_{i, c}^{2}\right)}{i_{\text {stop }}-i_{\text {start }}+1},
$$

where $\min _{c}(\cdot)$ is the minimum phase difference over all 4 channels, and $i_{\text {start }}$ and $i_{\text {stop }}$ specify the range bins of interest over which the radar system is set up to detect movement.

In addition to detecting small movements, we propose a second detection parameter, less sensitive to small changes in the environment. In this case, the radar studies the mean squared difference of the FFT magnitude to sense movements greater than the signal's wavelength $\lambda$. Mathematically, this can be presented as

$$
\mathcal{A}_{\mathrm{MS}}=\frac{\sum_{i=i_{\text {start }}}^{i_{\text {stop }}} \Delta \mathcal{A}_{i}^{2}}{i_{\text {stop }}-i_{\text {start }}+1},
$$

where $\Delta \mathcal{A}_{i}$ is the difference between two consecutive amplitudes at range bin $i$, and $i_{\text {start }}$ and $i_{\text {stop }}$ specify the range bins of interest.

\section{EXPERIMENTAL RESULTS}

To demonstrate the radar's capabilities, the system parameters are set as detailed in Table I. With $250 \mathrm{MHz}$ bandwidth, the system achieves a theoretical range resolution of $0.6 \mathrm{~m}$. Each pulse is a sequence of $4 \times 125$ 


\begin{tabular}{|c|c|}
\hline System Parameters & Value \\
\hline \hline Pulse duration: $\tau$ & $250 \mu \mathrm{s}$ \\
\hline Bandwidth: $\beta$ & $250 \mathrm{MHz}$ \\
\hline Range resolution: $\Delta R$ & $0.6 \mathrm{~m}$ \\
\hline Sampling frequency: $f_{s}$ & $500 \mathrm{kHz}$ \\
\hline Max. range: $R_{\max }$ & $37.5 \mathrm{~m}$ \\
\hline Samples per pulse: $L$ & 125 \\
\hline FFT size: & 512 \\
\hline Pulses per scan: $N$ & 16 (for slow-time proc.) \\
\hline PRI: $T_{\text {PRI }}$ & $1.5 \mathrm{~ms}$ \\
\hline Max. Doppler: $V_{\max }$ & $2.07 \mathrm{~m} \cdot \mathrm{s}^{-1} \approx 7.46 \mathrm{~km} \cdot \mathrm{h}^{-1}$ \\
\hline Doppler resolution: $\Delta V$ & $0.26 \mathrm{~m} \cdot \mathrm{s}^{-1} \approx 0.93 \mathrm{~km} \cdot \mathrm{h}^{-1}$ \\
\hline
\end{tabular}

TABLE I

LIST OF THE SYSTEM PARAMETERS AND THEIR VALUES.

snapshots sampled every $2 \mu \mathrm{s}$. Using this software configuration, the maximum unambiguous detection range is $37.5 \mathrm{~m}$. In the following use cases, the radar device is connected to a laptop through USB for both power and data transmission. The received data is parsed, plotted, and then stored for later use. To illustrate the radar's real-time performance, additional videos and raw data outputs are publicly available on the following link [27].

\section{A. Range-Direction Map}

This use case studies the practical range resolution of the developed radar. Fig. 8 describes a scenario where two persons are standing $1.2 \mathrm{~m}$ apart in front of an extended glass wall located at $19 \mathrm{~m}$. To reduce the sidelobes, a Hamming window applied over all 125 data samples.

Although the first target is at $7.3 \mathrm{~m}$, the signal's spectral density is still considerably high at close ranges. This is caused by insufficient isolation between the transmitter and receiver. Moreover, the $1.2 \mathrm{~m}$ gap between the test subjects corresponds to the experimental radar resolution.

In addition to the plot of FFT amplitudes, the radar also outputs a range-direction 3D plot. The bottom left graph in Fig. 8 presents the raw range-direction information computed by the micro-controller. As each range bin contains a single direction value, the final plot is a scatter of discontinuous dots. To obtain a continuous graph, the python code performs an additional smoothing operation. It averages the received data matrix with shifted version of itself, first across the angle dimension then the range dimension as detailed below:

1) Initialize $\mathbf{S}$ as the raw data matrix.
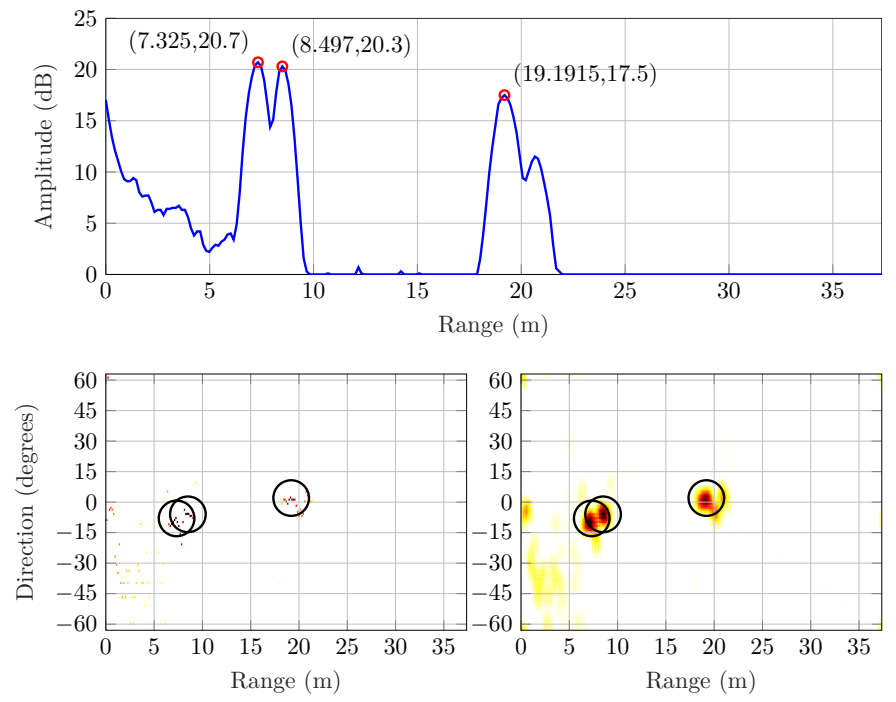

Fig. 8. Spectral density of a received signal (top) and its corresponding range-direction maps (bottom). Two people are standing in front of the radar. The first person is at $7.325 \mathrm{~m}$ while the second is $1.2 \mathrm{~m}$ farther. An extended glass wall is at $19 \mathrm{~m}$.

2) for $j=1,2, \cdots$, Shift $_{\mathrm{ANGLE}}$, evaluate

$$
\mathbf{S}=\mathbf{S}+\underbrace{\left[\mathbf{S}_{j+1: N,:}^{T} ; \mathbf{S}_{1: j,::}\right]}_{\mathbf{S} \text { shifted up }}+\underbrace{\left[\mathbf{S}_{N-j+1: N,:} ; \mathbf{S}_{1: N-j,:}\right]}_{\mathbf{S} \text { shifted down }} .
$$

3) for $i=1,2, \cdots$, Shift $_{\text {RANGE }}$, evaluate

$$
\mathbf{S}=\mathbf{S}+\underbrace{\left[\mathbf{S}_{:, i+1: M}, \mathbf{S}_{:, 1: i}\right]}_{\mathbf{S} \text { shifted left }}+\underbrace{\left[\mathbf{S}_{:, M-i+1: M}, \mathbf{S}_{:, 1: M-i}\right]}_{\mathbf{S} \text { shifted right }} .
$$

4) Normalize the smoothed matrix $\mathbf{S}$

This post-processing operation results in a smoother matrix presented in the bottom right plot of Fig. 8. It is recommended to shift the received raw matrix first along the angle axis to generate additional points per range bin and obtain smoother mainlobes. Here, the angle and range shifts are set to $\mathrm{Shift}_{\mathrm{ANGLE}}=3$ and $\mathrm{Shift}_{\mathrm{RANGE}}=2$ respectively.

\section{B. Measurement to Target Association}

The aim of the next experiment is to assess the performance of the measurement-to-target association algorithm. To this end, the radar is strapped to a user's chest who faces a wall and a cantilever. The user walks towards the wall and returns to his initial location. Next, a person enters the scene and walks back and forth in front of the radar [27].

The instantaneous range measurements of the closest three targets are plotted in Fig. 9 and compared to the output of the log table. In the top figure, the blue scatter correspond to the first target while the red and black dots correspond to the second and third detected 

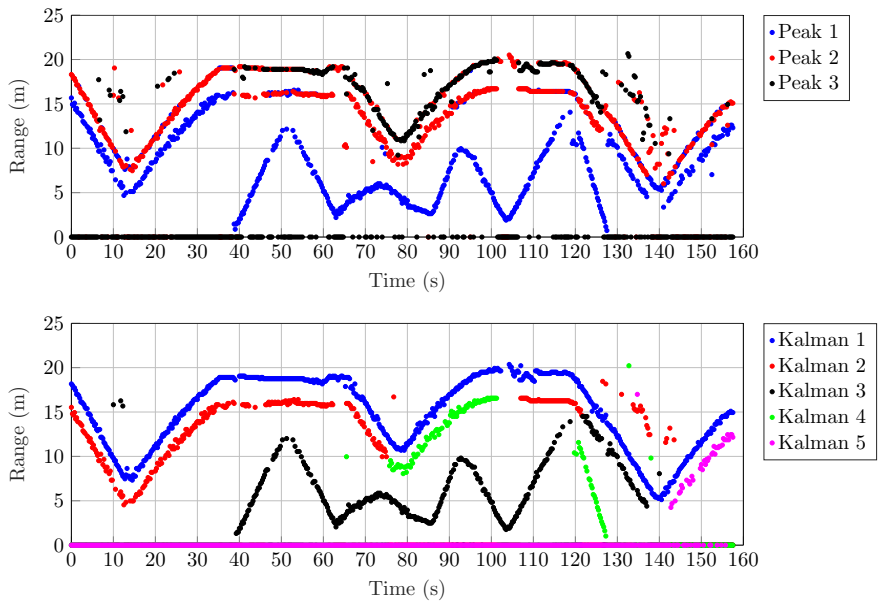

Fig. 9. Comparison between the instantaneous peak information of the three closest targets (top) and the output of the log table (bottom).

\begin{tabular}{|c|c|}
\hline Logging Method Parameters & Value \\
\hline \hline Max. guardband: $\Delta_{\mathrm{G}}$ & 15 bins $\approx 2.2 \mathrm{~m}$ \\
\hline Num. of occurrences: $N_{\mathrm{OC}} / N_{\mathrm{T}}$ & $3 / 5$ or $5 / 10$ or $15 / 32$ \\
\hline Num. of misses: $N_{\mathrm{DROP}}$ & 12 misses $\approx 3 \mathrm{~s}$ \\
\hline Min. distance: $\Delta_{\min }$ & 5 bins $\approx 0.75 \mathrm{~m}$ \\
\hline
\end{tabular}

TABLE II

PARAMETERS OF THE MEASUREMENT TO TARGET ASSOCIATION ALGORITHM.

peaks respectively. The upper plot in Fig. 9 shows that the instantaneous range information is not stable. The radar occasionally misses the first target, i.e., the walking person, and the blue dot lands on the background wall instead. Thus, the instantaneous information cannot directly feed the Kalman filter.

The lower plot in Fig. 9 illustrates the output of the measurement-to-peak association method. It shows that the measurements are grouped into multiple stable tracks. The instantaneous peaks are assigned to Kalman filters in a chronological order. For instance, the first detected target is linked to the first available Kalman filter. At instant $T=75 \mathrm{~s}$, the cantilever (i.e. second red target) is temporarily masked by the close test subject. Its range measurements became very noisy and fell outside the guard band. As a result, a new Kalman filter process is started. Again, at $T=100 \mathrm{~s}$, the cantilever is not detected for more than 3 seconds. Hence, it is dropped from the log table. When it is detected again, the measurements are assigned to the second Kalman index as it is the first available filter. At $T=120 \mathrm{~s}$, the walking subject stands directly below the cantilever. Since both targets are very close in range, the two tracks are merged. Later, a new track is created when the subject walks
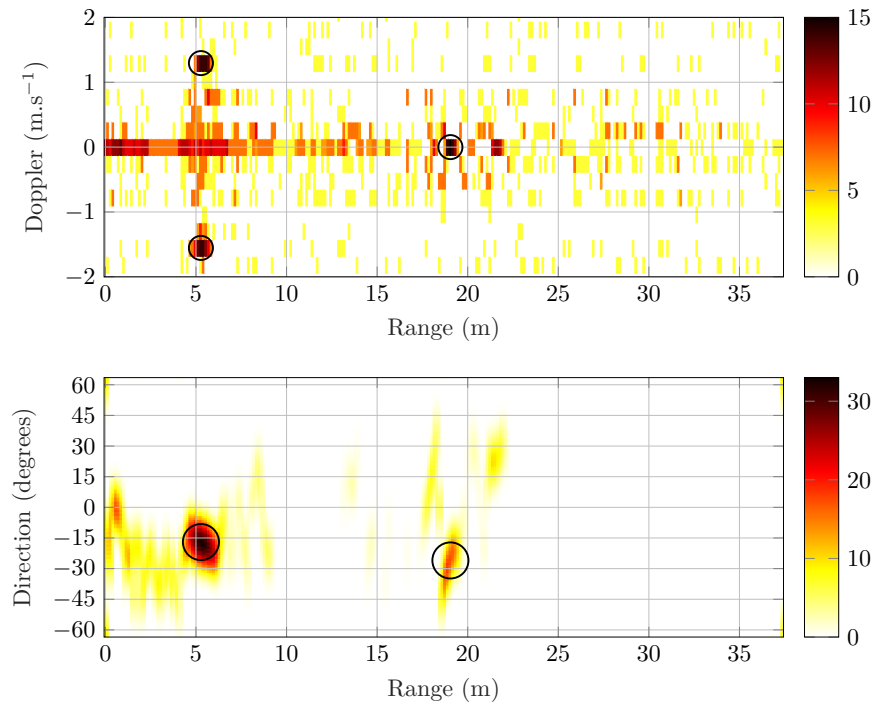

Fig. 10. Slow time processing output computed by the microcontroller in real time. The lower plot presents the range direction map of the same scene.

back towards the radar, i.e away from the cantilever. At each scan, the updated $T$ tracks feed their corresponding Kalman filters to smooth and track the rapidly changing range and direction information.

\section{Range-Doppler Map}

In this subsection, the performance of the RangeDoppler map is assessed. The slow time processing algorithm is activated and the radar transmits a sweep of 16 pulses. The goal is to estimate the velocity of a walking person. As the maximum velocity does not exceed $7 \mathrm{~km} \cdot \mathrm{h}^{-1} \approx 2 \mathrm{~m} \cdot \mathrm{s}^{-1}$, the pulse repetition interval is set to $1.5 \mathrm{~ms}$.

For this use-case, the experiment is similar to the one presented in Fig. 8 except that the subjects are now walking in opposite directions. The upper plot in Fig. 10 presents the result of the second FFT operation computed in real-time inside the micro-controller. The lower plot corresponds to the range direction map. When the two subjects pass next to each other, the range direction map shows only one target. Nonetheless, thanks to the Doppler information, the 2 targets are well separated. At range $5 \mathrm{~m}$, the targets are moving with opposite velocities -1.5 and $1.3 \mathrm{~m} \cdot \mathrm{s}^{-1}$. Therefore, the Doppler processing provides extra information which helps resolve two moving targets occupying the same range bin.

\section{Presence Detection and Vital Sign (breathing) Mon- itoring}

This subsection discusses the performance of the radar's third mode of operation: movement and res- 

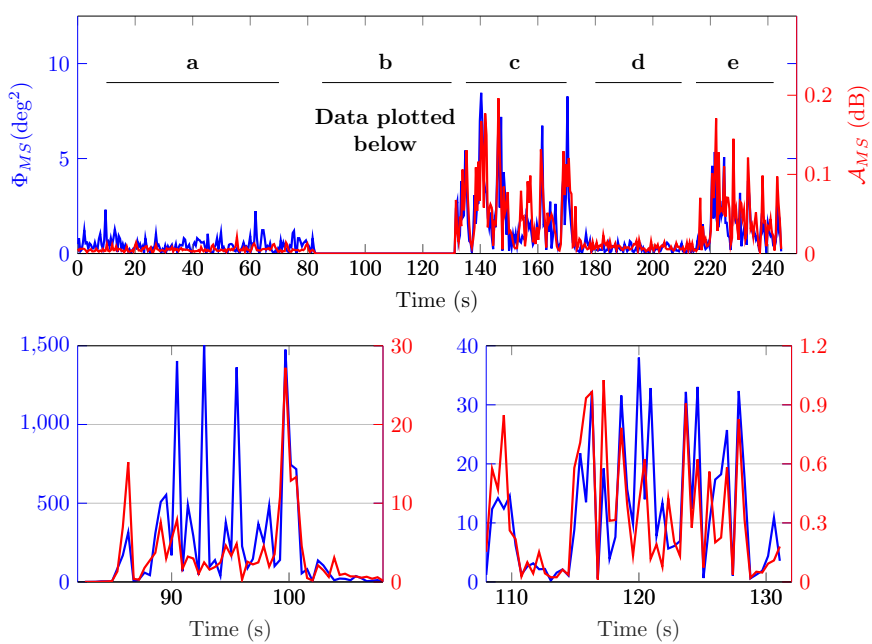

Fig. 11. Behavior of the phase based $\Phi_{M S}$ and amplitude based $\mathcal{A}_{M S}$ presence indicators for numerous scenarios: a- Empty room, b1- Person walking inside the room (bottom left) b2- Person moving his hands while seated (bottom right) c- Person breathing while seated d- Person holding his breath and e- Person breathing again

piration detection. For this purpose, the experiment is conducted inside a $4 \times 4 \mathrm{~m}^{2}$ room. The radar is located in the corner facing the entrance door, and a chair is in the diagonally opposing corner. To detect movements inside the room, the bins of interests are set from 20 to 45. It corresponds to the range from 3 to $6.5 \mathrm{~m}$. Hence, the radar is able to detect the chair and its immediate sidelobes.

Fig. 11 presents the behavior of the indicators $\Phi_{M S}$ and $\mathcal{A}_{M S}$ in various settings. In zone (a), the room is empty. When the subject enters, the indicators reach their highest values. They are separately plotted in the bottom left graph to avoid masking the smaller values. Afterwards, the subject walks around the room, sits on the chair and starts moving his hands. This setting is plotted in the bottom right graph. Next, the subject holds still while breathing normally (zone (c)). Afterwards, the person holds his breath for $50 \mathrm{~s}$ (zone (d)), then breathes again (zone(e)).

Table III lists the average value of the indicators during each setting. The $\mathcal{A}_{M S}$ average value is significant when the test subject walks around the room, and negligible in the remaining scenarios. It is mainly used to detect movements higher than the wavelength. Comparatively, the $\Phi_{M S}$ average changes noticeably from one scenario to another. In fact, it jumps from 0.3 to 2 depending on whether the target is breathing or not. This indicator is able to detect movements that are in the scale of the wavelength.

\begin{tabular}{|l|c|c|}
\hline Scenario & Average $\Phi_{M S}$ & Average $\mathcal{A}_{M S}$ \\
\hline \hline Empty room & 0.36 & $4.9 \times 10^{-3}$ \\
\hline $\begin{array}{l}\text { Person walking in- } \\
\text { side the room }\end{array}$ & 242.5 & 3.334 \\
\hline $\begin{array}{l}\text { Person moving while } \\
\text { seated on a chair }\end{array}$ & 11.56 & 0.323 \\
\hline $\begin{array}{l}\text { Person not moving } \\
\text { and just breathing }\end{array}$ & 2.11 & $56.9 \times 10^{-3}$ \\
\hline $\begin{array}{l}\text { Person not moving } \\
\text { and not breathing }\end{array}$ & 0.37 & $9.1 \times 10^{-3}$ \\
\hline
\end{tabular}

TABLE III

Average Values of THE $\Phi_{M S}$ AND $\mathcal{A}_{M S}$ INDiCATORS FOR EACH SCENARIO.

\section{CONCLUSION}

We described the hardware design of a $24 \mathrm{GHz}$ FMCW radar system suitable for surveillance, obstacle avoidance, speed estimation, and health care applications. We detailed the implemented signal processing algorithms that help detect moving and stationary targets in ranges up to $37.5 \mathrm{~m}$. The extended experimental results illustrated the capabilities of the proposed radar. With $250 \mathrm{MHz}$ bandwidth, the experimental range resolution is approximatively $1.2 \mathrm{~m}$. With slow time processing, the Doppler information is employed to resolve close targets moving with different velocities. The device can detect and track a walking person at $12 \mathrm{~m}$. The detection range can reach $20 \mathrm{~m}$ if the target's cross section is higher. When discussing the radar performance for presence detection and vital sign monitoring applications, we concluded that the phase based indicator $\Phi_{M S}$ is suitable for sensing small movements while the amplitude indicator $\mathcal{A}_{M S}$ detects large changes in the environment.

\section{REFERENCES}

[1] T. S. Rappaport, S. Sun, R. Mayzus, H. Zhao, Y. Azar, K. Wang, G. N. Wong, J. K. Schulz, M. Samimi, and F. Gutierrez, "Millimeter wave mobile communications for 5G cellular: It will work!," IEEE Access, vol. 1, pp. 335-349, 2013.

[2] M. Xiao, S. Mumtaz, Y. Huang, L. Dai, Y. Li, M. Matthaiou, G. K. Karagiannidis, E. Björnson, K. Yang, C. L. I, and A. Ghosh, "Millimeter wave communications for future mobile networks," IEEE Journal on Selected Areas in Communications, vol. 35, pp. 1909-1935, Sept. 2017.

[3] T. S. Rappaport, J. N. Murdock, and F. Gutierrez, "State of the art in 60-Ghz integrated circuits and systems for wireless communications," Proceedings of the IEEE, vol. 99, pp. 13901436, Aug. 2011.

[4] P. Adhikari, "Understanding millimeter wave wireless communication," Loea Corp.: White Paper, 2008.

[5] C. Iovescu and S. Rao, "The fundamentals of millimeter wave sensors," Texas Instruments: White Paper, May 2017. 
[6] R. Lachner, "Industrialization of mmwave sige technologies: Status, future requirements and challenges," in 2013 IEEE 13th Topical Meeting on Silicon Monolithic Integrated Circuits in RF Systems, pp. 105-107, Jan. 2013.

[7] W. Menzel, "Millimeter-wave radar for civil applications," in The 7th European Radar Conference, pp. 89-92, Sept. 2010.

[8] A. Zhu, X. Qi, T. Fan, Z. Gu, Q. Lv, D. Ye, J. Huangfu, Y. Sun, W. Zhu, and L. Ran, "Indoor localization for passive moving objects based on a redundant SIMO radar sensor," IEEE Journal on Emerging and Selected Topics in Circuits and Systems, vol. PP, no. 99, pp. 1-9, 2018.

[9] C. Li, V. M. Lubecke, O. Boric-Lubecke, and J. Lin, "A review on recent advances in Doppler radar sensors for noncontact healthcare monitoring," IEEE Transactions on Microwave Theory and Techniques, vol. 61, pp. 2046-2060, May 2013.

[10] T. Sakamoto, P. J. Aubry, S. Okumura, H. Taki, T. Sato, and A. G. Yarovoy, "Noncontact measurement of the instantaneous heart rate in a multi-person scenario using X-band array radar and adaptive array processing," IEEE Journal on Emerging and Selected Topics in Circuits and Systems, vol. PP, no. 99, pp. 114, 2018.

[11] H. Hong, L. Zhang, C. Gu, Y. Li, G. Zhou, and X. Zhu, "Noncontact sleep stage estimation using a CW doppler radar," IEEE Journal on Emerging and Selected Topics in Circuits and Systems, vol. PP, no. 99, pp. 1-11, 2018.

[12] G. M. Brooker, "Understanding millimetre wave FMCW radars," in 1 st International Conference on Sensing Technology, IEEE, New Zealand, pp. 152-157, Nov. 2005.

[13] E. Hyun, Y.-S. Jin, and J.-H. Lee, "Design and implementation of $24 \mathrm{GHz}$ multichannel FMCW surveillance radar with a software-reconfigurable baseband," Journal of Sensors, vol. 2017, 2017. Hindawi Publishing Corporation.

[14] P. Molchanov, S. Gupta, K. Kim, and K. Pulli, "Short-range FMCW monopulse radar for hand-gesture sensing," in IEEE Radar Conference, pp. 1491-1496, May 2015.

[15] "Guide sense." Url: http://www.guidesense.com/en/. Accessed: Mar. 18, 2018.

[16] T. Kiuru, M. Metso, M. Utriainen, K. Metsävainio, H.-M. Jauhonen, R. Rajala, R. Savenius, M. Ström, T.-N. Jylhä, R. Juntunen, and J. Sylberg, "Assistive device for orientation and mobility of the visually impaired based on millimeter wave radar technology-clinical investigation results," Cogent Engineering, vol. 5, no. 1, p. 1450322, 2018.

[17] Infineon, "BGT24MTR12, Silicon germanium $24 \mathrm{GHz}$ transceiver MMIC," BGT24MTR12 datasheet, p. 8, July 2014. Revision 3.2.

[18] D. Banerjee, Pll Performance, Simulation and Design. Dog Ear Publishing, 2006.

[19] "Analog Devices, ADIsimPLL." Url: https://form.analog.com/Form_Pages/RFComms/ADISimPll.aspx. Accessed: Jan. 4, 2018.

[20] D. G. Fang, Antenna Theory and Microstrip Antennas. Boca Raton, FL, USA: CRC Press, Inc., 1st ed., 2009.

[21] R. A. Sainati, CAD of Microstrip Antennas for Wireless Applications. Norwood, MA, USA: Artech House, Inc., 1996.

[22] M. Richards, Fundamentals of Radar Signal Processing. McGraw-Hill, 2005.

[23] G. L. Charvat, Small and Short-Range Radar Systems. Boca Raton, FL, USA: CRC Press, Inc., 1 ed., 2014.

[24] P. Gething, Radio Direction Finding and Superresolution. Electromagnetics and Radar Series, P. Peregrinus, 1991.

[25] B. Atayants, V. Davydochkin, V. Ezerskiy, V. Parshin, and S. Smolskiy, Precision FMCW Short-Range Radar for Industrial Applications:. Artech House, 2014.

[26] T. Kiuru, M. Metso, S. Jardak, P. Pursula, J. Häkli, M. Hirvonen, and R. Sepponen, "Movement and respiration detection using statistical properties of the fmcw radar signal," in 2016 Global Symposium on Millimeter Waves (GSMM) ESA Workshop on Millimetre-Wave Technology and Applications, pp. 1-4, June 2016.

[27] "FMCW gitlab Url: https://gitlab.com/MatlabFiles/FMCW_Radar. Accessed: Mar. 18, 2018. 